\title{
EXPLICIT CONTENT IMAGE DETECTION
}

\author{
Jorge Alberto Marcial Basilio ${ }^{1}$, Gualberto Aguilar Torres ${ }^{1}$, Gabriel Sánchez \\ Pérez ${ }^{1}$, Linda Karina Toscano Medina ${ }^{1}$, Héctor Manuel Pérez Meana ${ }^{1}$, Enrique \\ Escamilla Hernadez ${ }^{1}$ \\ ${ }^{1}$ Graduate School, Instituto Politécnico Nacional ESIME Culhuacan \\ Avenida Santa Ana \#1000 Col. San Francisco Culhuacan, Deleg. Coyoacán C.P. 04430, \\ México D.F. \\ ${ }^{1}$ \{jmarcialb0300, gaguilar, gsanchezp, ltoscano, hmperezm, eescamillah\} \\ dipn.mx
}

\section{ABSTRACT}

This paper proposes a system gives for explicit content image detection based on Computer Vision Algorithms, pattern recognition and FTK software Explicit Image Detection. In the first stage, HSV color model is used for the input images for the purpose of discriminating elements that are not human skin images. Then the image is filtered using skin detection. The output image only contains the areas of which it is composed. The results show a comparison between the proposed system and the company software Access Data called Forensic Toolkit 3.1 Explicit Image Detection isperformed.

\section{KEYWORDS}

Skin Detection, HSV Color Model, Explicit Content, Pattern Recognition, Computer Vision

\section{INTRODUCTION}

With the development of Internet, dramatically falling costs of data storage and advances in coding technology are generating a dazzling array of photography, animation, graphics sound and video [1]. Nowadays it is easy to have access to a computer with an Internet connection where there is currently a large amount of adult images for free downloading. This kind of media is also available for children and is an increasingly problem for many parents.

Filtering images with adult classified content is very important for searching principal Internet browser programs to avoid offensive content. Nowadays there are some ways to stop pornographic images on computers, such as blocking unwanted sites or identifying images that show explicit content. There are some programs in the foreign market that allow blocking sites on Internet with offensive or explicit content such as: CyberPatrol, ContentProtect, NetNanny, Family.net and K9 Web Protection [2].All these programs provide parental control to safeguard their children using the Internet. There are some others programs which detect pornographic images within the computer such as: SurfRecon that offers a program for this purpose, and despite being a tool of computer forensic, helps to detect images with explicit content.

The name of this tool is FTK Explicit Image Detection, which comes in the "FTK 3.1"version. There are some papers on this subject such as: the paper carried out by Forsyth and Fleck who designed software to detect naked people [3], Wiederhold and Wang design an algorithm for recognition of images with doubtful content [4], and Li Chen et al design a skin detector based-on Neural Network [5].

There are some investigators who carried out papers about adult image detection as: Xiaoyin Wang et al. [6] who proposed an algorithm to detect adult images, Yue Wang et al. [7] who proposed a way to help the algorithms to detect objectionable images using nipple detection, Huicheng Zheng et al. [8] designed a filtering system to adult images, Wonil Kim et al. [9] design

DOI : $10.5121 /$ sipij.2010.1205 
a neural network based adult image classification, Jiann-Shu Lee et al.[10]proposed an algorithm to naked image detection based on adaptive and extensible skin color model.

In this paper a new algorithm to detect explicit images is proposed. It is based on Computer Vision algorithms and pattern recognition techniques. First the images are changed from the color model to discriminate objects in the image of no interest. In the next part of the proposed system the image is filtered using skin detection, with the aim to segment a person or people within the image. Then we can estimate the probability of the image as an image with explicit content, by counting all pixels with some skin tone.

The paper is organized as follows. An introduction of color models RGB and HSV, and the representation of skin detector used for the system are presented in the section 3 and 4 respectively. Section 4 presents a brief description of the proposed system, and in section 5 are the results. Finally the conclusions are given.

\section{COLOR MODELS}

\subsection{RGB Color Model}

The RGB color model is an additive color model in which the primary colors red, green, and blue light are added together in various ways to reproduce a broad array of colors. The name comes from the initials of the three colors Red, Green, and Blue. The RGB color model is shown in the Figure 1.

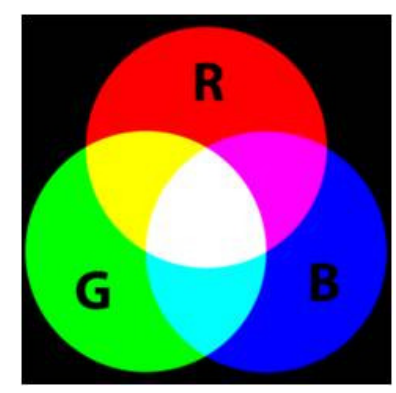

Figure 1. RGB Color Model

The main purpose of the RGB color model is for sensing, representation, and display of images in electronic systems, such as televisions and computers.

The RGB color model is an additive in the sense that three light beams are added together to make a final color. To form a color with RGB, three colored light beams (one red, one green, and one blue) should be superimposed. Each of the three beams is called a component of that color, and each can have arbitrary intensity, from fully off to fully on, in the mixture. Zero intensity for each component gives the darkest color (no light, considered the black), and full intensity of each gives a white.

A color in the RGB color model is described by indicating how much of each of the red, green, and blue is included in each component which can vary from zero to a defined maximum value which depends of the application. In computing, the component values are often stored as integer numbers in the range 0 to 255 . 


\subsection{HSV Color Model}

HSV color model (Hue, Saturation, and Value) is a no lineal transformation of the RGB space color, and the colors are a combination of the three values: the Hue $(\mathrm{H})$, Saturation or color quantity $(\mathrm{S})$, and itself value $(\mathrm{V})$. These values are represented in a circular diagram, as shown in Figure 2 .

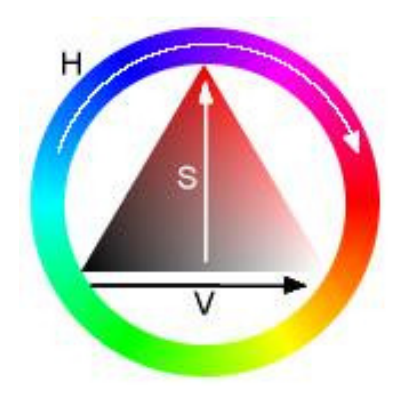

Figure 2. HSV Color Model

The three magnitudes can have the following values

- Hue: The type of color (e.g. red, green, or yellow). These are represented as a degree of angle whose possible values range from 0 to $360^{\circ}$ (although for some applications are normalized from 0 to $100 \%$ ).

- Saturation: Is represented as the distance from the axis of the black-white glow. The possible values range from 0 to $100 \%$.

- Value: Represents the height in the black-white axis. The possible values range from 0 to $100 \% .0$ is always black. Depending on the saturation, 100 could be white or a more or less saturated color.

Using this color model as an input image is converted using the mathematical expressions (1) to (3) that are shown below.

$$
\begin{aligned}
& H=\arccos \frac{1 / 2[(R-G)+(R-B)]}{\sqrt{\left.(R-G)^{2}+(R-B)(G-B)\right]}} \\
& S=1-3 \frac{\min (R, G, B)}{R+G+B} \\
& \quad V=\frac{1}{3}(R+G+B)(3)
\end{aligned}
$$

Once the transformation of the input image was made, it was observed that the skin tone of a person could be seen in a different color from those seen from different objects within the same image. An example of this is shown in Figure 3, which is a sample of conversion to the HSV color model of an image in RGB color model. 
Signal \& Image Processing : An International Journal(SIPIJ) Vol.1, No.2, December 2010

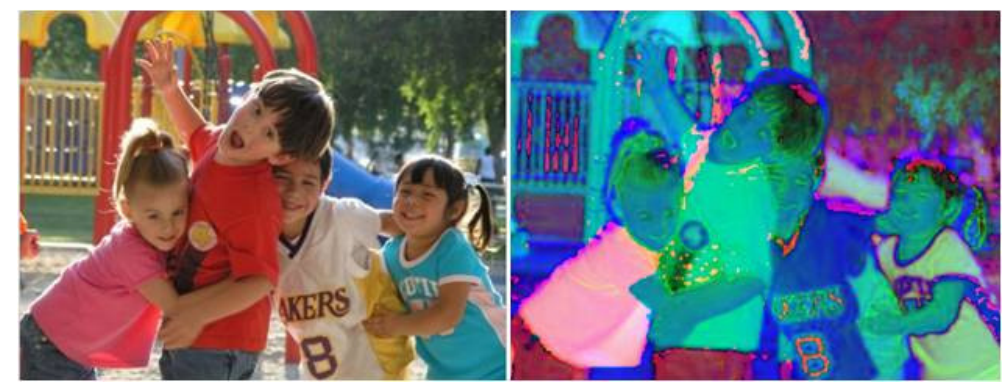

(a)

(b)

Figure 3. (a) RGB Image (b) HSV Image

As mentioned, an advantage of using this particular color model is that we can rule out many objects using a simple filter, in this case we use skin detection to get only the areas of skin which are the most important for our objective.

\section{SKIN DETECTION}

Skin detection can help detect a human limb, torso, or face within a picture. Lately many methods of skin identification within a digital image have been developed. Skin color has proved to be a useful and robust method for face detection, localization and tracking. There have been a number of researchers who have looked at using color information to detect skin. Jones and Rehg [11] constructed a color model using histogram-learning techniques at RGBcolor space. Yang and Auhuja [12] estimated probability density function of human skin color using a finite Gaussian mixture model whose parameters are estimated through the EM algorithm. There are other researchers who have developed papers about the different models of skin detection as Vezhnevets et al. [13], Kakumanu et al.[14], Kelly et al. [15].

In this paper a novel solution using the HSV color model, which is very similar to the RGB color model, is proposed.

Once the change of color model has been made, the next stage is to proceed to pixel detection with human skin. This was achieved by observing several images, which are a threshold where most people with different skin color within the image can be segmented.

To determine the threshold it was necessary to make an analysis of the histograms in the HSV color model. As observed in Figure 4 an image of the face of a girl identified with major clarity the threshold that we need. 


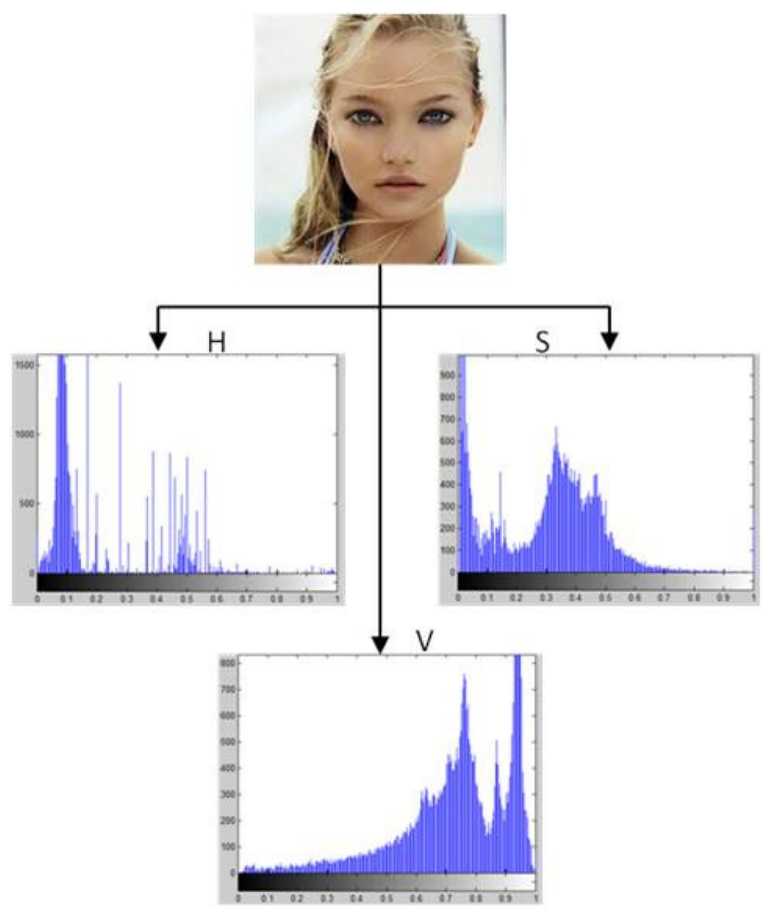

Figure 4. Histograms of HSV Color Model

The histograms observed in Figure 4 helps to have an idea of the values, which could be taken to choose a threshold able to take the skin values. This would be correct if only detecting people with the same skin color of the girl used as reference is desired but in Internet there exists a large amount of images that not only contain people with a specific skin color, but also people with different skin color, so after exhaustive analysis the threshold decided was the following:

$$
\begin{aligned}
& H>0 \text { and } H<0.25 \\
& S>0.15 \text { and } S<0.9 \\
& V>0.2 \text { and } V<0.95
\end{aligned}
$$

Where $\mathrm{H}, \mathrm{S}, \mathrm{V}$ are in the range from 0 to 1.

With the purpose of finding naked people, there are other kinds of features such as the percentage of pixels detecting similar skin color. Based on these features, a procedure of segmentation is carried out in color images. Some examples are illustrated in Figure 5 which shows people with different skin color and can be seen that the threshold used works appropriately.

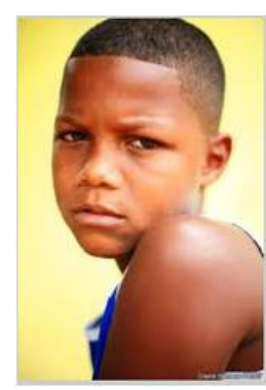

(a)

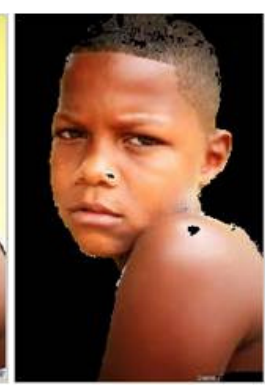

(b) 


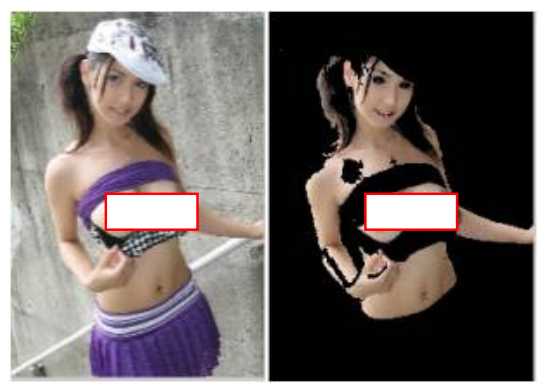

(c)

(d)

Figure 5. Skin Color Segmentation

(a) and (c) Original Images, (b) and (d) Segmented Images

The proposed threshold in this section was able to detect skin color zones effectively.

The method proposed for skin detection can find skin areas, but to decide which of a comprehensive set of images contain naked people is still a great challenge.

\section{Proposed System}

In the Figure 6 shows the proposed system, which has one part: a classifier.

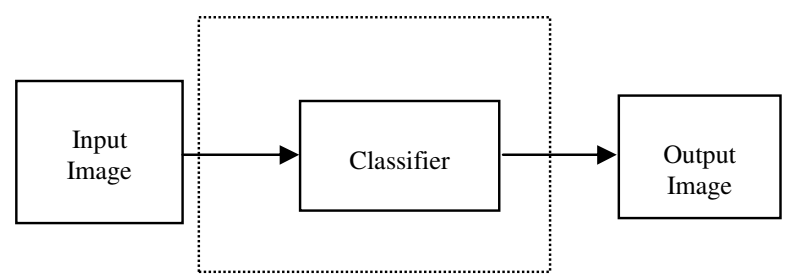

Figure 6.Proposed System

The proposed system is created a classifier, using the HSV color model, which allows us to recognize texture within the image, and this way can detect skin to know if there are pixel areas with some skin tone, and the value of the likelihood that the input image was an image with explicit content can be estimated.

Skin detection can be used as the basis for detection of the images with explicit content because there is a considerable relationship between the images with large areas of skin and pornographic images or with explicit content.

\section{ReSUltS}

A test to prove the performance of the algorithm was used using different images from Internet. The system can process different kind of images, as images in different lighting conditions and images with different size.

The input images for testing are classified in: a) images of naked people or with explicit content and b) natural images. In the images with explicit content people Asians, Caucasian, Europeans, Latin Americans and a little amount of people with black skin can be found. In natural images there are different kinds of images such as: dressed people, animals, plants, cars, cartoons, landscapes and others were also obtained from Internet. 
First the input image is transformed from the RGB to the HSV color model. Next stage using the threshold that is proposed in section 3 the image is filtered usingthe skin detection to identify the areas that contain some skin color, and in this way only get the image of the person or people within the input image.

At this point, all skin areas detected are taken, and proceed to count the amount of pixels that there are within the image to estimate the likelihood that this image is classified as an image with explicit content or not. The results obtained are shown in Table 1.

To be able determine if the input image has explicit content, a mathematic expression is proposed, described in (4), and in this way obtain the percentage of amount of skin that there are in the input image. If the percentage is more than $50 \%$ it is considered to have objectionable content.

$$
\text { skin percentage }=\frac{\# \text { skin color pixels }}{\text { \#image pixels in total }} \times 100
$$

Table 1. Classification results using the proposed system

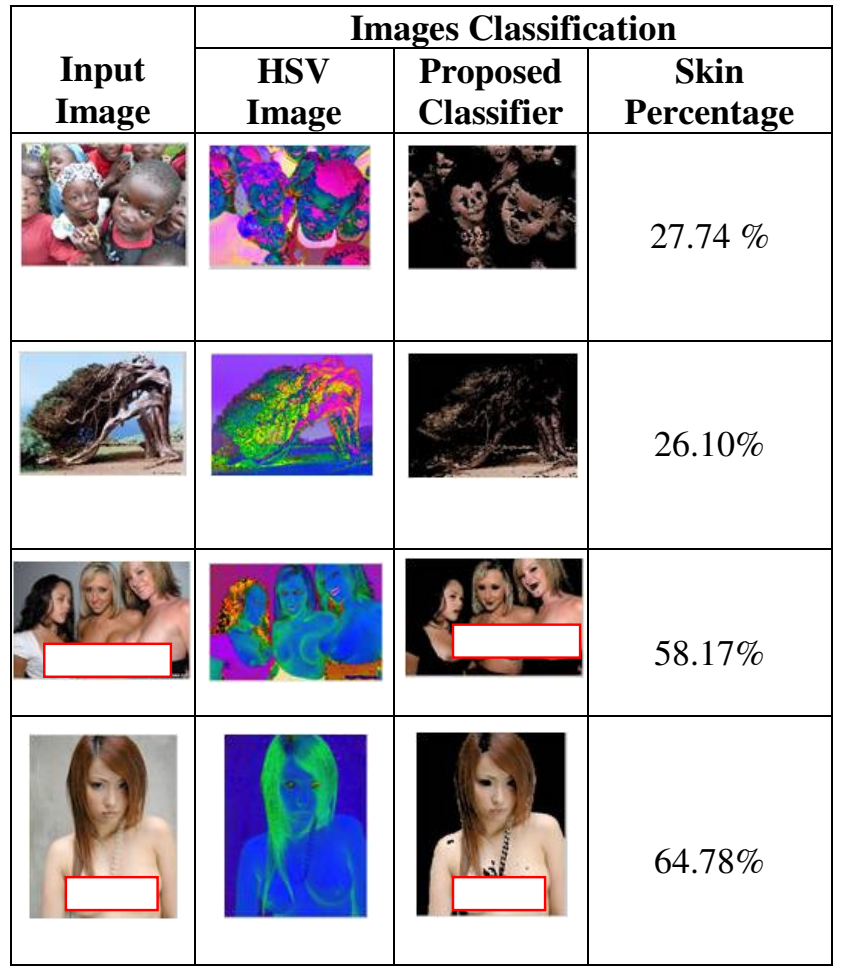

The Table 1 shows four images of the set, which the proposed system was proved, in this results can be observed that the third and the fourth image are classified as an image with explicit content.

This system is used as reference to know if the analyzed images have a certain quantity of pixels with skin color. This is a factor to determine if the image has naked people, as the majority of images that have undressed people are made up of skin zones that take up most of the image. 
Signal \& Image Processing : An International Journal(SIPIJ) Vol.1, No.2, December 2010

Within the results, it can be observed that the selected threshold for the image segmentation works efficiently in people with white skin, although to brown people or black people, this threshold does not segment the total of skin areas. However, we can estimate its naked people likelihood with the fact that details are not lost detecting such things.

Also software was used from Access Data called Forensic Toolkit 3.1, to make the comparison between the proposed system and its system to detect images. It makes a forensic image of the device that have to analyze, after the analysis began with three algorithms to determine the percentage of explicit content of an image. The description of the three algorithms used for evaluate the images was obtained from [16] and was shown in the Table 2:

Table 2. Explicit Image Detection Profile Types

\begin{tabular}{|c|c|l|}
\hline $\begin{array}{c}\text { Profile } \\
\text { Name }\end{array}$ & Level & \multicolumn{1}{|c|}{ Description } \\
\hline X- DFT & Default (XS1) & This is the most generally accurate, It is always selected. \\
\hline X-FST & Fast (XTB) & $\begin{array}{l}\text { This is the fastest. It scores a folder by the number of files it } \\
\text { contains that meets the criteria for a high likelihood of } \\
\text { explicit material. } \\
\text { It is built on a different technology than X-DFT and does } \\
\text { not use "regular" DNAs. It is designed for very high } \\
\text { volumes, or real-time page scoring. Its purpose is to quickly } \\
\text { reduce, or filter, the volume of data to a meaningful set. }\end{array}$ \\
\hline X-ZFN & $\begin{array}{c}\text { Less False } \\
\text { Negatives (XT2) }\end{array}$ & $\begin{array}{l}\text { This is a profile similar to S-FST but with more features and } \\
\text { with fewer false negatives than X-DFT. } \\
\text { Apply this filter after initial processing to all evidence, or to } \\
\text { only the folders that score highly using the X-FST option. } \\
\text { Check-mark or highlight those folders to isolate them for } \\
\text { Additional Analysis. }\end{array}$ \\
\hline
\end{tabular}

To test its system, the same set of input images was used using the three profiles that have. The results of the software are shown in the Table 3. 
Signal \& Image Processing : An International Journal(SIPIJ) Vol.1, No.2, December 2010

Table 3. Results obtained with FTK 3.1

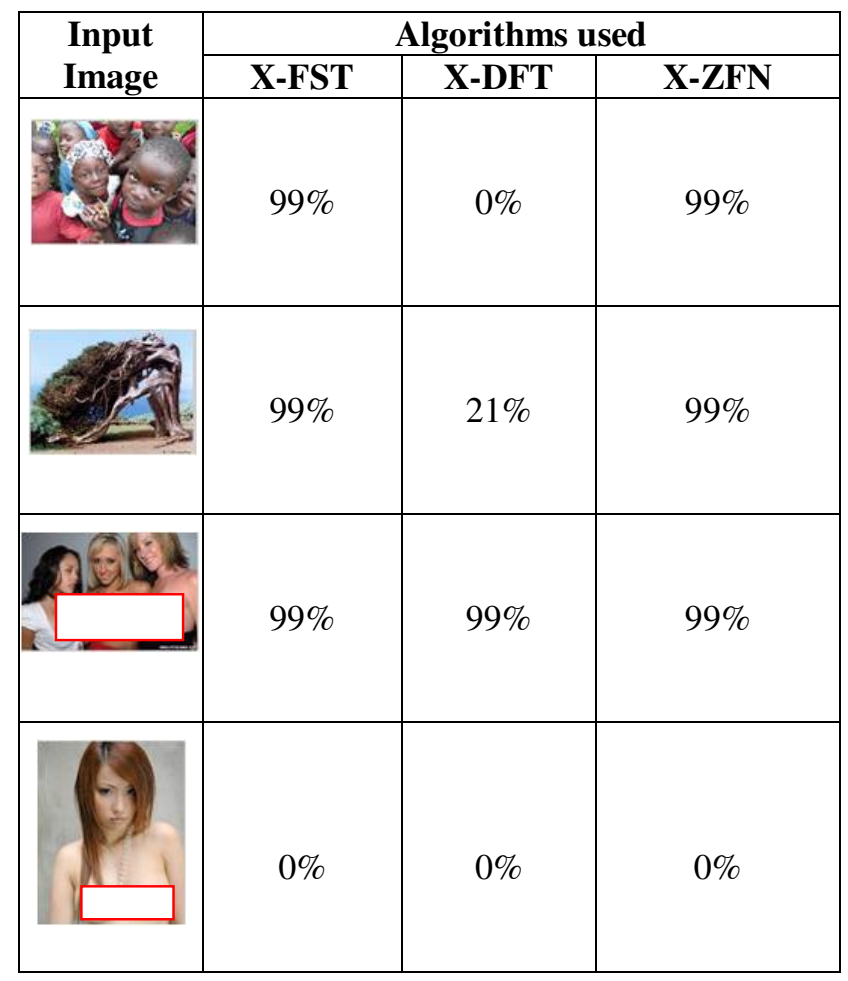

The software from Access Data is used only to help the users take decisions whether the images have or lack explicit content, as can be observed in the Table 3. There is a discrepancy between the percentage that gives the result and the content of original image.

\section{Conclusions}

This paper proposed an algorithm to detect images with explicit content in color images, using the HSV color model and a method of skin detection which works effectively although in some images it could find some errors, due to the image lighting conditions when taken, another factor that can be by a bad interpretation of the system.

HSV color model is an important method to be able to decrease all the lighting problems that the image could be had; moreover, using this color model is more visible the skin tone than the RGB color model, for this reason is used HSV color model to be able to do skin detection.

The proposed system gives an output image that only shows color skin pixels within the image, in basis to this can be known the likelihood that the image is an explicit content image or not, due to explicit content image in most part has color skin pixels.

The importance of the comparison between the proposed system and the software Forensic Toolkit 3.1 was done to know if the proposed system could do the same work, and this way know whether the input image is an explicit content image or not, at final could prove that the system proposed carry out effectively. 
Signal \& Image Processing : An International Journal(SIPIJ) Vol.1, No.2, December 2010

\section{REFERENCES}

[1] L. Duan, G. Cui, W. Gao and H. Zhang, “Adult Image Detection Method Base-on Skin Color Model and Support Vector Machine", The $5^{\text {th }}$ Asian Conference on Computer Vision, 23-25 January 2002, Melbourne, Australia.

[2] C. Habis, and F. Krsmanovic, "Explicit Image Filter,” Stanford Univ., 2005.

[3] D. A. Forsyth. M. Fleek, and C. Bregler, "Finding naked people", Proc.Forth European Conference on Computer Vision. pp 593-602, 1996.

[4] G. Wiederhold and J. Z. Wang, "WIPE (TM): Wavelet Image Pornography Elimination; A System for Screening Objectionable Images".

[5] Li Chen, J. Zhou, Z. Liu, W. Chen and G. Xiong, "A Skin Detector Based on Neural Network”, In IEEE 2002 International Conference on Communication, Circuits and Systems and West Sino Expositions, vol. 1, 615 - 619, 2002.

[6] Xiaoyin Wang, Changzhen Hu, and Shuping Yao, "An Adult Image Recognizing Algorithm Based on Naked Body Detection”, In Computing, Communication, Control, and Management, 2009. CCCM 2009. ISECS International Colloquium on, 197 - 200, 2009.

[7] Yue Wang, Jun Li, HeeLin Wang, and ZuJun Hou, “Automatic Nipple Detection Using Shape and Statistical Skin Color Information”, Advances In Multimedia Modeling, LNCS, Vol. 5916/2010, $644-649,2010$.

[8] Huicheng Zheng, Hongmei Liu, and Daoudi, M, "Blocking Objectionable Images: Adult Images and Harmful Symbols”, In. Multimedia and Expo, 2004. ICME '04. 2004 IEEE International Conference on, Vol. 2, 1223 - 1226, 2004.

[9] Wonil Kim, Han-Ku Lee, Seong Joon Yoo and Sung Wook Baik, "Neural NetworkBased Adult Image Classification”, Artificial Neural Networks: Biological Inspirations - ICANN 2005 LNCS, Volume 3696/2005, 481 - 486, 2005.

[10] Jiann-Shu Lee, Yung-Ming Kuo, Pau-Choo Chung, and E-Liang Chen, “An adult image Identification System Employing Image Retrieval Technique", In. Pattern Recognition Letters, Vol. 28, 2367 - 2374, 2007.

[11] M. J. Jones and J. M. Regh, "Statistical color models with application to skin detection", In Proc. Of the CVPR '99, vol. 1, 274-280, 1999.

[12] M. H. Yang and N. Ahuja, "Detecting human faces in color images", In International Conference on Image Processing (ICIP), vol 1, 127-130, 1998.

[13] V. Vezhnevets, V. Sazonov, A. Andreeva, “A Survey on Pixel-based Skin Color Detection Techniques”, In Proceedings of the GrapiCon, 85-92, 2003.

[14] P. Kakumanu, S. Makrogiannis, N. Bourbakis, “A survey of skin-color modeling and detection methods", In Pattern Recognition, Vol. 40, pp. 1106-1122, 2007.

[15] W. Kelly, A. Donnellan and D. Molloy, "Screening for Objectionable Images: A Review of Skin Detection Techniques", In Machine Vision and Image Processing Conference (IMVIP '08), 151 158, 2008.

[16] FTK 3.1 User Guide. 


\section{Authors}

Jorge Alberto Marcial-Basilio received the BS degree on Electronic and Communications Engineer in 2003 from the National Polytechnic Institute of Mexico. Actually he is a Master degree student at the Mechanical and Electrical Engineering School of the National Polytechnic Institute of Mexico.

Gualberto Aguilar-Torres received the BS degree on Electronic andCommunications Engineer; and The MS degree on MicroelectronicEngineering, in 2003 and 2005, respectively, from the National Polytechnic Institute of, Mexico. In 2005 he received the Best Thesis award from the National PolytechnicInstitute of Mexico for his Master research work and a memberof the National Researchers System of Mexico.

Gabriel Sanchez-Perez received the BS degree on Computer ScienceEngineer; and the $\mathrm{PhD}$ degree on Electronic and Communications, in 1999 and 2005, respectively, from the NationalPolytechnic Institute, Mexico City.From January 2001 to October 2006 he joined the Computer EngineeringDepartment Electrical and Mechanical Engineering School at the NationalPolytechnic Institute as Assistant Professor. In October 2006 he joints theGraduate School of the National Polytechnic Institute of Mexico where he isnow a Professor. Prof. Sanchez-Perez is a member of the IEEE.

Linda Karina Toscano-Medina received the BS degree on Computer Science Engineer; and the $\mathrm{PhD}$ degree on Electronic and Communications, in 1999 and 2005, respectively, from the National Polytechnic Institute, Mexico City. From January 2003 to October 2007 she joined the Computer Engineering Department Electrical and Mechanical Engineering School at theNational Polytechnic Institute as Assistant Professor. In October 2007 shejoints the Graduate School ofthe National Polytechnic Institute of Mexico where she is now a Professor. Prof. Toscano Medina is a member of theNational Researchers System of Mexico
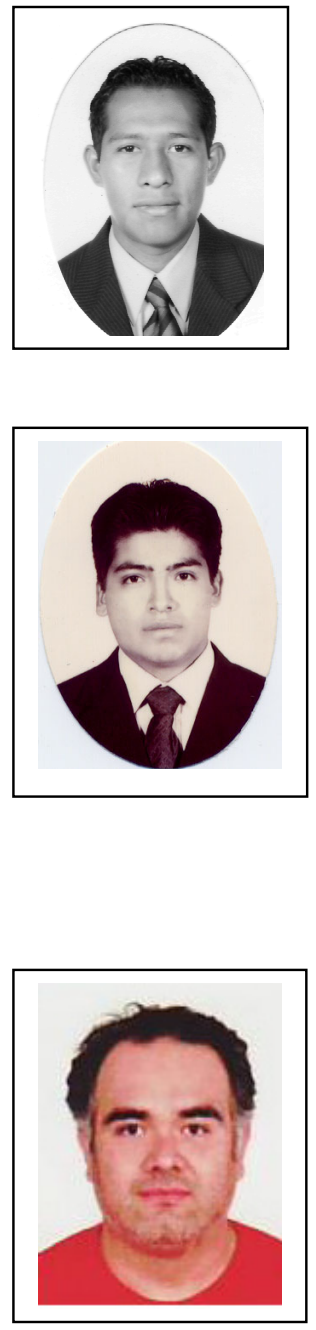
Signal \& Image Processing : An International Journal(SIPIJ) Vol.1, No.2, December 2010

Hector Perez-Meana received the BS Degreein Electronics Engineers from the UniversidadAutonoma Metropolitana (UAM) MexicoCity in 1981, the M.S. degree from the Universityof Electro-Communications, Tokyo Japan inMarch 1986, and a Ph. D. degree in ElectricalEngineering from Tokyo Institute of Technology, Tokyo, Japan, in 1989. In 1981 hejoined the Electrical Engineering Department ofthe Metropolitan University where he was a Professor.From March 1989 to September 1991,he was a visiting researcher at Fujitsu Laboratories Ltd, Kawasaki, Japan.In February 1997, he joined the Graduate Department of The Mechanicaland Electrical Engineering School on the National Polytechnic Institute ofMexico, where he is now a Professor. In 1991 Prof. Perez-Meana receivedthe IEICE excellent Paper Award, and in 1999 and 2000 the IPN ResearchAward. In 1998 Prof.Perez-Meana was Co-Chair of the ISITA'98. Hisprincipal research interests are signal and image processing, pattern recognition,watermarking, steganography and related fields. Dr. PerezMeana isa senior member of the IEEE, a member of the IEICE, the IET, the NationalResearchers System of Mexico and the Mexican Academy of Science.

Enrique Escamilla-Hernandez was born in Hidalgo, Mexico, on November 15th, 1973. He received the B.Sc. degree in Electronic from Metropolitan Autonomous University, DF, Mexico, in 1998 and the M.Sc. degree in microelectronic sciences from IPN, D.F., Mexico, in 2003 and Ph.D. degree in communication and electronic from IPN, D.F., Mexico, in 2006. His research interests are in designing algorithms for robust and intelligible image and speech signal processing for robot applications.
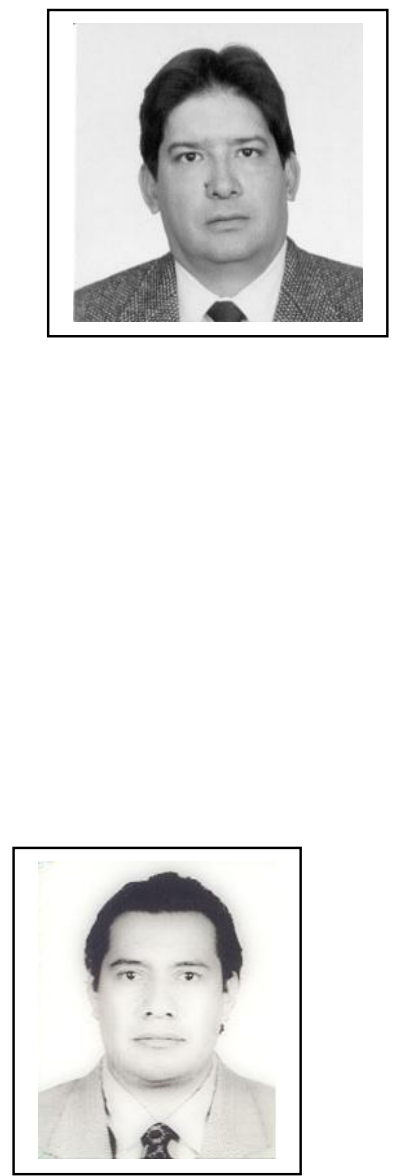